\title{
The Evolution of Dieulafoy's Lesion Since 1897: Then and Now-A Journey through the Lens of a Pediatric Lesion with Literature Review
}

\author{
Jenna-Lynn Senger and Rani Kanthan \\ Department of Pathology and Laboratory Medicine, Royal University Hospital, \\ University of Saskatchewan, Saskatoon SK, Canada S7N 0W8 \\ Correspondence should be addressed to Rani Kanthan, rani.kanthan@saskatoonhealthregion.ca \\ Received 10 September 2011; Accepted 7 November 2011 \\ Academic Editor: Cataldo Doria
}

Copyright ( $) 2012$ J.-L. Senger and R. Kanthan. This is an open access article distributed under the Creative Commons Attribution License, which permits unrestricted use, distribution, and reproduction in any medium, provided the original work is properly cited.

Background. In 1897, Dieulafoy was the first to characterize a gaping arteriole within the gastric mucosa causing massive hematemesis, designating it as "exulceratio simplex." A hundred years later, this vascular abnormality, now commonly referred to as a "Dieulafoy lesion," has been identified through the entire gastrointestinal tract and the bronchus. Objectives and Methods. As the original findings have been subjected to revisions and modifications by modern authors, Dieulafoy's original paper was reviewed and analyzed. The evolution of the current usage of "Dieulafoy's lesion" in the literature has been summarized with comparisons to the original report. Additionally, an index case of a 10-year-old female with a gastric "exulceratio simplex" is reported with a review of previously reported paediatric Dieulafoy lesions. Conclusions. The term "Dieulafoy lesion" in modern literature no longer adheres to the initial conclusions with regards to its origin, demographics, and presenting symptoms. Dieulafoy lesions remain a rare cause of gastrointestinal bleeding that can cause life-threatening haemorrhages in children.

\section{Introduction}

Paul Georges Dieulafoy (1839-1911), a professor of pathology at the Faculty of Medicine in Paris, France, was the first to describe a series of 10 patients who presented with massive hematemesis (approximately $4 \mathrm{~L}$ of blood in less than 24 hours) due to a bleeding gastric vessel, without any evidence of ulceration in the first three lessons of the 1897-1898 edition of "Clinique Médicale de l'Hôtel-Dieu." At autopsy, a superficial ulceration with a gaping arteriole was found in the gastric submucosa. The lesion's borders were not hardened or projecting, and the remainder of the mucosa was in perfect health. Dieulafoy concluded that this lesion was not a typical gastric ulcer and named it an "exulceratio simplex," which in time became known as a "Dieulafoy lesion" [1].

Modern literature has broadened the original definition of a "Dieulafoy lesion." Initially only described in the stomach, such findings have been additionally reported in the rest of the gastrointestinal tract such as the esophagus
[2], duodenum [3], ileum [4], jejunum [5], colon [6], anal canal [7], and rectum [7, 8]. Dieulafoy lesions have also been reported as a case series in nongastrointestinal sites such as the bronchus [9]. The advent of endoscopy has drastically changed the process of diagnosing and treating Dieulafoy lesions, with techniques such as endoscopic laparoscopic banding (ELB), thermocoagulation, and injections with adrenaline/epinephrine (EPI) as alternatives to replace surgical management as was described in the mid-1800s as bestpractice management.

\section{Materials and Methods}

2.1. Objective. Dieulafoy's original treatise published in French was reviewed, analyzed, and translated into English for discussion as we suspected that the original findings have been subjected to revision with modification by many current authors. Using an index case of a 10-year-old female who presented with recurrent upper gastrointestinal bleeding 
that necessitated a partial gastrectomy, the pathological abnormality of the vascular anomaly in the submucosa"Dieulafoy lesion"-is analyzed from a historical viewpoint of "then and now." All published paediatric cases of Dieulafoy lesions as available in PubMed and Medline have also been retrieved and analyzed providing a comprehensive review of paediatric Dieulafoy lesions.

Part 1: Revisiting the Past. We obtained a copy of the original first three lessons of "Clinique Médicale de l'Hôtel-Dieu" treatise written in 1897-1898 by Paul Georges Dieulafoy. The original French paper was read and analyzed in its native language and translated into English for detailed review and study.

Part 2: Analyzing the Present. The definition and usage of a "Dieulafoy lesion" in modern literature was explored. The most widely recognized definition of a "Dieulafoy Lesion" was retrieved from Wikipedia, an extensively accessed free online encyclopaedia. We then researched the definition, epidemiology/aetiology, clinical presentation, detection, and treatment of this lesion using Google Scholar, PubMed, and Medline. Finally, a search of PubMed and Medline limited to the paediatric population (ages 0-18) was conducted in the English and French languages. All references were read, reviewed, and analyzed.

Part 3: Moving Forward: a New Paediatric Index Case. A 10-year-old female presented to the Emergency Room with melena, hematemesis, and generalized abdominal pain. At presentation, her haemoglobin was $6.5 \mathrm{~g} / \mathrm{dL}$ with a blood pressure of 99/64. Gastroscopy identified four polypoid lesions on the lesser curvature surrounded by an erythematous and friable mucosa that was oozing blood. Multiple mucosal biopsies showed no significant pathological abnormality. Imaging including an MRI showed no significant findings, and a repeat gastroscopy was unremarkable. She was discharged home on prevacid.

A year and half later, she was admitted to the paediatric intensive care unit (PICU) after vomiting approximately $1 \mathrm{~L}$ of bright red blood preceded by a one-day history of general malaise and fatigue. She was hypotensive at presentation, with a systolic pressure of $70 \mathrm{mmHg}$ and haemoglobin of $7.5 \mathrm{~g} / \mathrm{dL}$. She was stabilized by transfusion with fluids and two units of packed red blood cells (PRBCs). The abdominal ultrasound was normal. A repeat gastroscopy rediscovered the $4-5 \mathrm{~cm}$ region of erythematous and friable gastric mucosa and raised the possibility of an underlying vascular abnormality. A second repeat MRI revealed diffuse thickening of the greater curvature with several enlarged mesenteric lymph nodes; the largest measured 5-6 mm.

On laparoscopic examination, prominent, tortuous vessels were seen on the lateral aspect of the greater curvature and extensively on the posterior wall of the stomach. The greater omentum inhibited a full visualization, and it was decided to carry out an exploratory laparotomy to determine the full extent of this vascular abnormality. At intraoperative examination, mild handling of the stomach caused extensive bleeding, which required vascular clamps and two units of

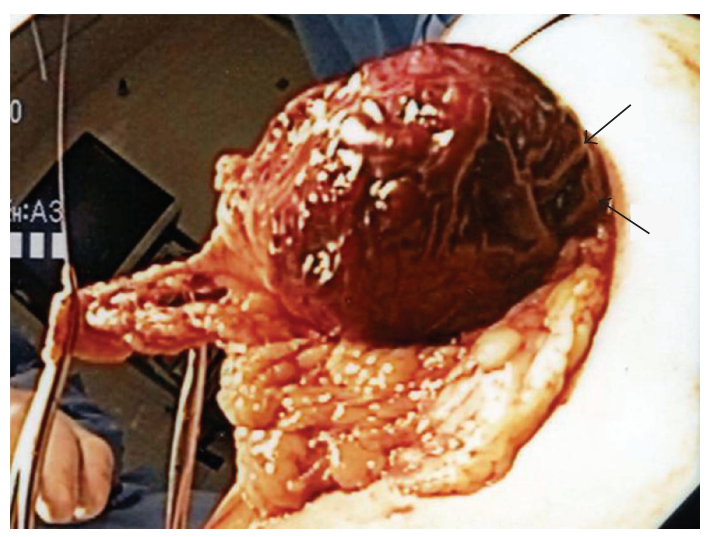

FIGURE 1: Intraoperative specimen of stomach at partial gastrectomy showing the rich vascular abnormality seen within the posterior gastric wall (arrow).

PRBCs to maintain haemostasis. No "normal" gastric wall was visible on the posterior aspect (Figure 1) resulting in the decision to proceed with a partial gastrectomy to include the anterolateral and posterior walls of the stomach.

Histopathological examination of the specimen showed a hemorrhagic area measuring $3.3 \times 2.2 \mathrm{~cm}$ with a central $0.4 \times 0.4 \mathrm{~cm}$ vascular polyp. On microscopic examination, a large dilated tortuous vessel with evidence of fibrin thrombi and recent haemorrhage through the overlying mucosa was identified (Figures 2(a) and 2(b)). This submucosal vessel measured up to $8.3 \mathrm{~mm}$ in some sections. No accompanying vein was identified. The mucosa appeared congested with pseudopolypoid regions, and there was no evidence of inflammation or significant erosion. Special stain demonstrated the presence of elastin in the wall of the persistent large vessel confirming its arterial origin (Figures $2(\mathrm{c})$ and $2(\mathrm{~d})$ ). Additional sections also showed the presence of complex anastomosing submucosal vessels with mucosal and submucosal evidence of interstitial haemorrhage. The diagnosis of a Dieulafoy's lesion in the proximal stomach was confirmed.

Postoperative recovery was uneventful. As she had no further complaints at her followup at the outpatient clinic 6 weeks later, her care has now been transferred to her family physician.

All information provided in this paper has been completely deidentified and is thus exempted from a formal review by the Ethics Committee as per our institutional policy.

\section{Results and Discussion}

3.1. Then: The Original Dieulafoy Lesion. Though in 1884 Gallard wrote the first description of a patient with a "Dieulafoy's lesion" [10], this eponym is attributed to the publication of Clinique Médicale de l'Hôtel-Dieu de Paris written by Paul Georges Dieulafoy. He was the first to characterize in detail a lesion he called "exulceratio simplex." In the first three lessons of this treatise, Dieulafoy describes 10 cases of exulceratios and notes that the features that differentiate these lesions from ulcers are caused by tuberculosis, syphilis, typhoid fever, uremia, and alcoholism. 


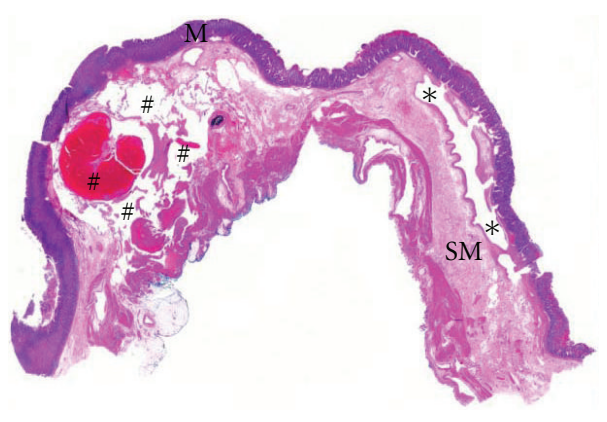

(a)

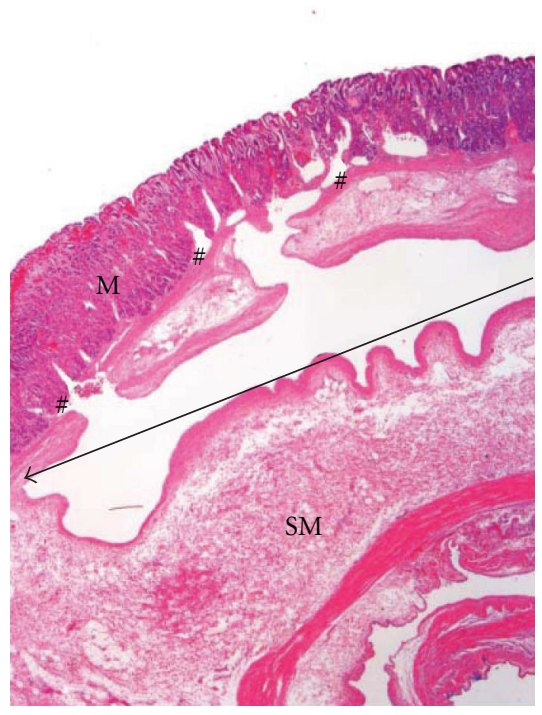

(c)

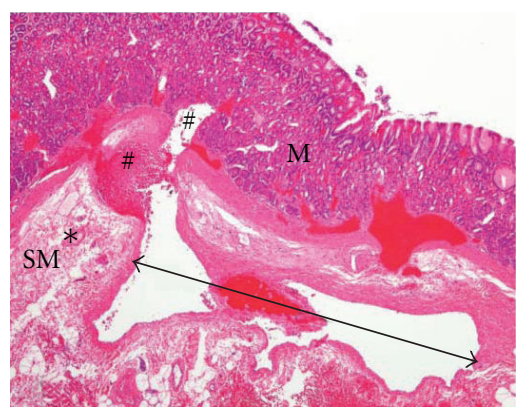

(b)

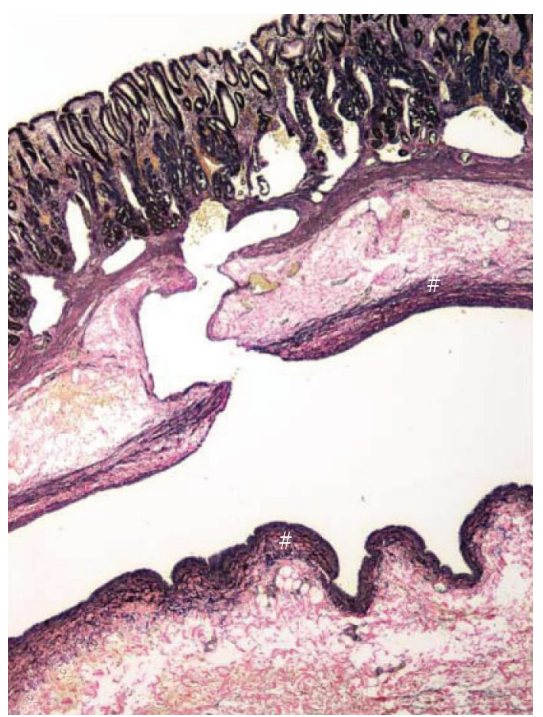

(d)

FIGURE 2: Histopathological findings. (a) Whole-mount scan of hematoxylin and eosin stained slide showing the exulceratio simplex $(*)$ in the submucosa (SM) (below the mucosa (M)) as described by Dieulafoy. Additionally, (\#) shows the presence of a complex rich anastomosis of varying sized vessels in the submucosa with an intraluminal haemorrhagic thrombus (cirsoid aneurysm of gastric vessels). (b) Low magnification (objective lens $\times 2$ ) of hematoxylin and eosin-stained slides shows the presence of blood within the large tortuous, caliberpersistent artery () in the submucosa (SM) with evidence of rupture and haemorrhage (\#) through the overlying mucosa (M). A normalsized submucosal arteriole $(*)$ is seen at the same level. (c) High magnification (objective lens $\times 10$ ) of hematoxylin and eosin-stained slides shows the presence of the large caliber-persistent artery () in the submucosa (SM) —Dieulafoy's lesion—with communications (\#) into the overlying noninflamed mucosa $(\mathrm{M})$. (d) High magnification (objective lens $\times 10$ ) of elastin-stained slides shows the presence of elastin in the wall (\#) of the exulceratio simplex confirming its arterial origin.

Histological examination of the lesion revealed "sur l'une des petites érosions cratériformes, due à la disparition de la muscularis mucosae, apparaît làrtériole béante" ("on one of the small crateriform erosions, caused by disappearance of the muscularis mucosa, appears a gaping arteriole," page 4). $\mathrm{He}$ concludes the three lessons with eight major learning points about the "exulceratio simplex" (pages 42-43) [1]:

(1) the exulceratio simplex is "à l'estomac, une perte de substance très superficielle et assez étendue" ("in the stomach, a loss of substance very superficial and fairly extensive," Page 42);

(2) the "exulceratio simplex" is oval, elliptical, or star shaped and has the dimensions of a 50-centime piece, of 1 franc or 2 francs. It is "très superficielle et ne dépasse pas en profondeur la tunique muqueuse" ("very superficial and does not extend deeper than the tunica mucosa," page 42 );

(3) edges of the exulceratio "n'étant ni indurés ni surelevés" ("neither indurate nor raised," page 42) and the gastric walls maintain their softness;

(4) the exulceratio "est un processus ulcéreux aigu" ("is an acute ulcerative process," page 43) that reaches a voluminous and superficial submucosal arteriole;

(5) rarely the exulceratio simplex presents with gastric symptoms, but more often it evolves "silencieusement, sournoisement" ("silently, slyly," page 43) in a healthy patient who may develop nausea, vertigo, syncope, and large haematemeses;

(6) to diagnose exulceratio simplex, the physician must identify a "true" haematemesis and then "acquérir la 
certitude que la lesion ... siège bien à l'estomac" ("gain certainty that the lesion is sitting in the stomach," Page 43);

(7) the exulceratio simplex is extremely serious due primarily to the substantial haematemeses;

(8) the future risk of recurrence remains unknown.

3.2. Now: Dieulafoy Lesions Described in the Current Literature. In the current literature, alternative names, descriptions, and criteria for Dieulafoy lesions include "caliberpersistent artery," "gastric aneurysm," "gastric arteriosclerosis," "submucosal arteriole malformation," and "cirsoid aneurysm" $[11,12]$. The "exulceratio simplex" as described in 1897 is estimated to account for $0.5-14 \%$ of upper GI bleeding $[13,14]$. It is, however, suggested that a lack of recognition rather than true rarity may be responsible for this low incidence [15]. Most lesions (75-95\%) are in the proximal stomach, particularly within $6 \mathrm{~cm}$ of the gastroesophageal junction on the lesser curve [16], yet they have also been described in nongastric sites such as the duodenum [3], jejunum [5], ileum [17], rectum [8], and in nongastrointestinal sites such as the bronchus [9]. This clearly digresses from Dieulafoy's sixth learning point which states that the lesion "siège bien à lèstomac" ("sits in the stomach," page 43) [1]. Dieulafoy lesions are more common in men than women, at a $2: 1$ ratio [18] and the mean age at presentation is within the 5 th decade of life (range 50-70 years) [15]. This contrasts strongly with Dieulafoy's observation that "l'exulceratio à une predilection pour les jeunes gens" ("the exulceratio has a predilection for young people," page 36) [1].

Dieulafoy lesions classically present as intermittent and massive GI haemorrhage with symptoms associated with this blood loss. $44 \%$ of patients report melena, 30\% haematemesis, $18 \%$ with both hematemesis and melena, $6 \%$ with haematochezia, and 1\% with iron-deficiency anemia [17] with the mean reported haemoglobin at admission ranging between 8.4 and $9.2 \mathrm{~g} / \mathrm{dL}$ [13]. In Dieulafoy's original report, all patients presented with acute massive haematemesis such that his learning point no. 6 emphasizes the importance of accurate identification of haematemesis. He also stresses that "les hémorragies stomacales dues à léxulceratio simplex sont... pas annoncées par de petits vomissements de sang" ("gastric haemorrhages due to the exulceratio are not announced by little vomits of blood," page 32) [1]. In our index case, however, the acute massive bleed was preceded by "little vomits of blood" that occurred almost a year and a half prior. Perhaps due to the older age of onset, comorbidities are present in up to $90 \%$ of patients, the most common including ischemic heart disease, hypertension, diabetes mellitus, liver disease, and renal failure [12]. Interestingly Dieulafoy commented that patients with the "exulceratio simplex" "ressemblent à des gens bien portants" ("seemed like healthy people," page 41 and conclusion no. 5) [1].

Though over a hundred years have passed since Dieulafoy first described this lesion, the mechanisms causing the tortuosity and the persistence of the large-sized submucosal arteries remain unknown. Many reports have attributed this lesion to the pathological structure or the size of the artery with a persistent musculoelastic mantle of Wanke. Debate continues regarding the exact pathology and linkage of this artery to the overlying mucosa [35]. Further, what actually triggers their submucosal-to-mucosal rupture has yet to be proven. No family predisposition [3] or risk factors have been identified despite research that has searched for a link with nonsteroid anti-inflammatory drugs (NSAIDs), alcohol, tobacco, or a history of peptic ulcer disease [7]. The lesion's predominance on the lesser curve of the stomach may be explained by the vascular architecture of this region which arises directly from an arterial chain [15]. The most widely accepted theory which takes into account the paediatric cases suggests that the tortuous artery with an increased diameter and often a variable course length is a congenital anomaly [25]. As such, a large submucosal artery may be abnormally close to the mucosa, facilitating its emergence and making it more vulnerable to rupture [32]. All patients of the original series had only a single ruptured artery, leading Dieulafoy to note that "elle passerait assez facilement inaperçue sans un examen attentive et sans l'idée préconcue qu'on va la trouver" ("it would easily enough pass unperceived without a thorough examination and without the preconceived idea that we will find it," page 24) [1]. The theories of the cause of rupture are more variable, but it is agreed that something causing mucosal erosion or ischemic injury must be the initial step [17]. The three principal theories of pathogenesis include the following: (a) pulsations of the abnormally large artery may disrupt the mucosa and exposure of the artery to gastric/bowel contents which then chemically or mechanically erode and cause the bleed $[12,17]$, (b) gastric "wear and tear" promotes the formation of an arterial thrombus that causes necrosis [17], and (c) age-related atrophy [17].

The advent of endoscopy has marked a drastic change in the diagnostic evaluation and therapeutic management of Dieulafoy lesions. Up to $71 \%$ of initial endoscopies are diagnostic only if there is active haemorrhage of at least $0.5 \mathrm{~mL} / \mathrm{min}$ [15]. However, in $6 \%$ of patients, the intermittent nature of the bleeding often requires multiple endoscopies to establish a definitive diagnosis [12, 17]. Unsuccessful endoscopies in $44 \%$ of patients are due to the presence of excess of blood in the stomach/bowel, and the remaining $56 \%$ are subtle lesions that are often overlooked $[12,17]$. Because of this subtlety, concomitant lesions such as ulcers or varices may be incorrectly diagnosed as being responsible for the bleed [13]. To diagnose a Dieulafoy lesion, the endoscopic visual criteria that must be met include (a) active arterial spurting or oozing from a small $(<3 \mathrm{~mm})$ defect in the mucosa, (b) visualization of a vessel protruding from a slight defect or normal mucosa, and/or (c) a fresh blood clot adherent to a defect of normal mucosa as described by Dieulafoy $[1,16]$.

Should endoscopy fail to determine a source of bleeding, angiography and capsule endoscopy may provide useful information. On an angiogram, extravasation of contrast into the GI system from an eroded artery is indicative of this lesion, as is the presence of tortuous ectatic vessels in the arterial phase without early venous return thereby 


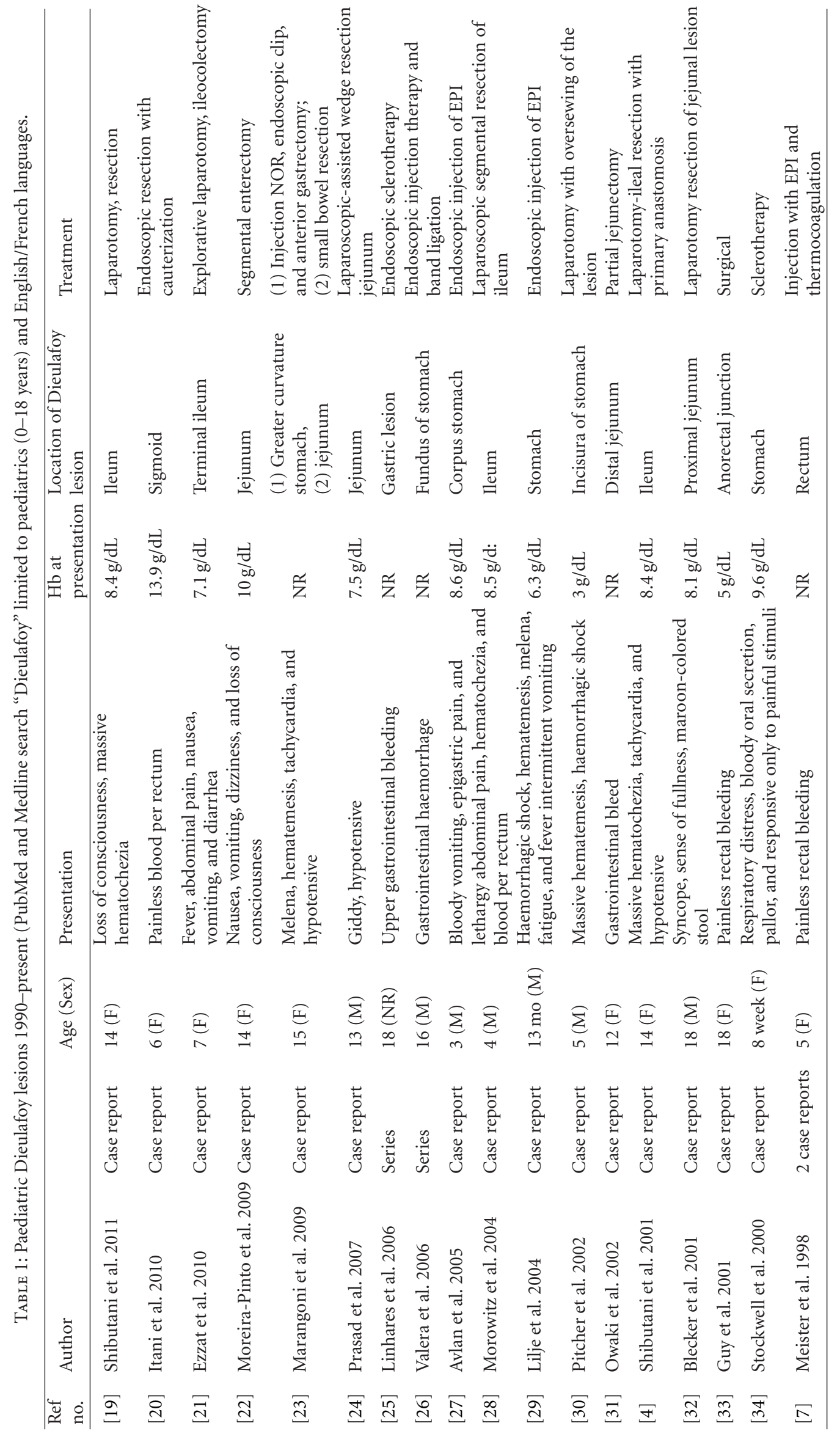


distinguishing this unique lesion from arteriovenous malformation [12]. This diagnostic modality is particularly useful for colorectal lesions where poor bowel preparation may obscure colonoscopy results [17]. A relatively new option, capsule endoscopy, is a noninvasive, direct way of investigating areas of the GI tract that are difficult to access by EGD or colonoscopy. There is a risk, however, of missing these small lesions if the camera is pointed to the wrong way and is of limited use in an emergency situation [17]. There is limited knowledge about the use of capsule endoscopy in children, as its size precludes its usefulness [17, 24].

Since 1986, endoscopy in addition to being the primary diagnostic tool has also become the first-line method of treatment [25]. Traditionally treated with a gastrotomy or gastrectomy, surgery is now reserved for the $4-8 \%$ of cases that do not achieve endoscopic haemostasis [36]. The three main forms of endoscopic treatment include (a) thermal electrocoagulation, heat probe coagulation, argon plasma coagulation, (b) regional injection-epinephrine (EPI) or norepinephrine (NOR) injection and sclerotherapy, and (c) mechanical banding and hemoclips $[17,32,36]$. A recent study by Alis et al. found that endoscopic band ligation (EBL) was associated with a significantly lower risk of recurrent bleeding as well as a shorter hospital stay when compared with sclerotherapy [36]. Monotherapy has a $9-40 \%$ higher risk of rebleeding versus combination therapy [17]. The site of the bleed is often tattooed with India ink for future identification in the event of a recurrent bleed [18]. The overall mortality rate of Dieulafoy lesions is $8.6 \%$, which is constant whether treated endoscopically or surgically [12]. The mainstay of surgical treatment for endoscopic failures is wide wedge resection or local excision such as partial/wedge gastrectomy [37]. Simple oversewing of the lesion is not recommended as it is associated with a greater risk of recurrent bleeding [37].

3.3. Pediatric Dieulafoy Lesions. Dieulafoy lesions as a cause of massive gastrointestinal bleeding are uncommon occurrences in the paediatric population. The most common cause of massive GI bleeding in children is usually related to oesophageal varices secondary to portal hypertension and chronic liver disease $[27,30]$. These "exulceratio simplex" are infrequent in children, and in his original description, Dieulafoy did not encounter any children with this rare form of gastrointestinal bleeding [1].

As listed in Table 1, twenty four cases of paediatric Dieulafoy lesions have been published in the English language as available in PubMed and Medline between 1990 and 2011 $[4,6,7,15,19-34,38-41]$. Though the reported age ranged from 8 weeks [34] to 18 years [25, 32, 33], children tended to be older, with the mean age of 10 years.

Unlike Dieulafoy lesions in the adult population, there is no clear preponderance of these lesions in pediatric males. Such strong male predominance in adults is perhaps hormonally related and can be attributed to the lack of estrogenic protective effects in males. It is also postulated that complex hormonal dysfunctions at the submucosal arteriole arcade are involved in the pathogenesis of the caliber- persistent artery as described by Dieulafoy's illustrations on pages 15 and $16[1]$.

The location of Dieulafoy lesions reported in children includes ten in the stomach, five in the jejunum, five in the ileum, and two in each the duodenum, and rectum and one in each of the sigmoid, anorectal junction, and anal canal. Twelve children (46\%) were treated surgically, a much higher percentage than in the adult population already described, while 14 were managed endoscopically by injection therapy, band ligation, and thermocoagulation (Table 1). Recently, minimally invasive diagnostic and therapeutic techniques such as capsule endoscopy assisted by laparoscopic resections, conventional bidirectional endoscopy, push enteroscopy, and radionuclide imaging with techniques such as multidetector-row computed tomography (MDCT) have opened new avenues in the management of obscure and occult gastrointestinal bleeding in children $[8,24]$. Further, GI bleeding in children continues to pose many different challenges to the clinician as there is no general consensus in the investigative and treatment protocols to be followed [24].

\section{Conclusion}

The usage of the term "Dieulafoy lesion" in the current literature no longer fits within the confines of the conclusions drawn by its namesake over a hundred years ago. Dissidents contest with Dieulafoy's original theory that the "exulceratio simplex" though of congenital origin represents an acute process. Other digressions now include an aging population, nongastric and extraintestinal locations, and its recognition in children as well as in adults. Though massive haematemesis-"gastrorrhagias"-was the sine qua non of the "original" Dieulafoy lesion, the inclusion of nongastric (oesophagus, duodenum, jejunum, ileum, colon, anal canal, and rectum) and extraintestinal (bronchus) sites of this anomaly has completely altered the clinical presentation. Additionally, endoscopic vascular criteria have replaced the diagnosis of this lesion in these widespread locations.

Nevertheless, the name "Dieulafoy lesion" is entrenched in the literature and contains historical significance; therefore, we suggest that this eponym may still be used with the understanding that the diversity of the lesion it describes no longer conforms to the original definition of the term. Dieulafoy lesions remain a rare and most dangerous form of gastrointestinal bleeding that can result in life-threatening haemorrhages in children.

\section{References}

[1] G. Dieulafoy, "Exulceratio simplex: leçons 1-3," in Clinique Médicale de l'Hotel Dieu de Paris, G. Dieulafoy, Ed., pp. 1-38, Masson et Cie, Paris, France, 1898.

[2] J. Thimmapuram, M. Shah, and J. Srour, "Esophageal Dieulafoy lesion: an unusual cause of GI bleeding," Gastrointestinal Endoscopy, vol. 73, no. 5, pp. 1055-1056, 2011.

[3] M. B. Ujiki, A. Adler, L. L. Swanstrom, T. S. Diwan, and P. D. Hansen, "Emergent pancreaticoduodenectomy for Dieulafoy 
lesion of the duodenum," American Surgeon, vol. 76, no. 6, pp. 656-657, 2010.

[4] S. Shibutani, H. Obara, S. Ono et al., "Dieulafoy lesion in the ileum of a child: a case report," Journal of Pediatric Surgery, vol. 56, no. 5, pp. 17-19, 2001.

[5] M. E. Nga, S. A. Buhari, P. T. Iau, and G. C. Raju, "Jejunal Dieulafoy lesion with massive lower intestinal bleeding," International Journal of Colorectal Disease, vol. 22, no. 11, pp. 1417-1418, 2007.

[6] N. M. Dy, C. J. Gostout, and R. K. Balm, "Bleeding from the endoscopically-identified Dieulafoy lesion of the proximal small intestine and colon," American Journal of Gastroenterology, vol. 90, no. 1, pp. 108-111, 1995.

[7] T. E. Meister, G. W. Varilek, L. S. Marsano, L. K. Gates, Y. Al-Tawil, and W. J. S. de Villiers, "Endoscopic management of rectal Dieulafoy-like lesions: a case series and review of literature," Gastrointestinal Endoscopy, vol. 48, no. 3, pp. 302305, 1998.

[8] Y. Y. Chen and H. H. Yen, "Massive bleeding from a rectal Dieulafoy lesion: combined multidetector-row ct diagnosis and endoscopic therapy," Surgical Laparoscopy, Endoscopy and Percutaneous Techniques, vol. 18, no. 4, pp. 398-399, 2008.

[9] A. Parrot, M. Antoine, A. Khalil et al., "Approach to diagnosis and pathological examination in bronchial Dieulafoy disease: a case series," Respiratory Research, vol. 9, p. 58, 2008.

[10] T. Gallard, "Aneurysmes miliaires de l'estomac donnant lieu à des hématémèses mortelles," Bulletins et Mémoires de la Société Médicale des Hôpitaux de Paris, vol. 1, pp. 84-91, 1884.

[11] S. K. Faes, B. R. Untch, C. Edwards, J. Turner, M. Poleski, and D. S. Tyler, "Management of Dieulafoy's lesions," Gastrointestinal Bleeding, vol. 1, pp. 31-37, 2010.

[12] G. Alshumrani and M. Almuaikeel, "Angiographic findings and endovascular embolization in Dieulafoy disease: a case report and literature review," Diagnostic and Interventional Radiology, vol. 12, no. 3, pp. 151-154, 2006.

[13] M. Njeru, A. Seifi, Z. Salam, and L. Ognibene, "Dieulafoy lesion: a rare cause of gastrointestinal bleeding," Southern Medical Journal, vol. 102, no. 3, pp. 336-337, 2009.

[14] W. Lim, T. O. Kim, S. B. Park et al., "Endoscopic treatment of dieulafoy lesions and risk factors for rebleeding," Korean Journal of Internal Medicine, vol. 24, no. 4, pp. 319-322, 2009.

[15] C. P. Driver and J. Bruce, "An unusual cause of massive gastric bleeding in a child," Journal of Pediatric Surgery, vol. 32, no. 12, pp. 1749-1750, 1997.

[16] W. Apiratpracha, J. K. Ho, J. J. Powell, and E. M. Yoshida, "Acute lower gastrointestinal bleeding from a dieulafoy lesion proximal to the anorectal junction post-orthotopic liver transplant," World Journal of Gastroenterology, vol. 12, no. 46, pp. 7547-7548, 2006.

[17] M. Baxter and E. H. Aly, "Dieulafoy's lesion: current trends in diagnosis and management," Annals of the Royal College of Surgeons of England, vol. 92, no. 7, pp. 548-554, 2010.

[18] R. Garg, "Bleeding from a gastric Dieulafoy lesion," Emergency Medicine Journal, vol. 24, no. 7, p. 520, 2007.

[19] S. Shibutani, H. Obara, S. Ono et al., "Dieulafoy lesion in the ileum of a child: a case report," Journal of Pediatric Surgery, vol. 46, no. 5, pp. E17-E19, 2011.

[20] M. Itani, T. Alsaied, L. Charafeddine, and N. Yazbeck, "Dieulafoy's lesion in children," Journal of Pediatric Gastroenterology and Nutrition, vol. 51, no. 5, pp. 672-674, 2010.

[21] R. F. Ezzat, H. A. Hussein, T. S. Baban, A. T. Rashid, and K. M. Abdullah, "Typhoid ulcer causing life-threatening bleeding from Dieulafoy's lesion of the ileum in a seven-year-old child: a case report," Journal of Medical Case Reports, vol. 4, article 171,2010 .

[22] J. Moreira-Pinto, C. Raposo, V. T. da Silva et al., "Jejunal Dieulafoy's lesion: case report and literature review," Pediatric Surgery International, vol. 25, no. 7, pp. 641-642, 2009.

[23] G. Marangoni, A. B. Cresswell, W. Faraj, H. Shaikh, and M. J. Bowles, "An uncommon cause of life-threatening gastrointestinal bleeding: 2 synchronous Dieulafoy lesions," Journal of Pediatric Surgery, vol. 44, no. 2, pp. 441-443, 2009.

[24] T. R. S. Prasad, H. L. Kwang, H. L. Kiat, and T. L. Yap, "Bleeding jejunal dieulafoy pseudopolyp: capsule endoscopic detection and laparoscopic-assisted resection," Journal of Laparoendoscopic and Advanced Surgical Techniques, vol. 17, no. 4, pp. 509-512, 2007.

[25] M. M. Linhares, B. H. Filho, V. Schraibman et al., "Dieulafoy lesion: endoscopic and surgical management," Surgical Laparoscopy, Endoscopy and Percutaneous Techniques, vol. 16, no. 1, pp. 1-3, 2006.

[26] J. M. Valera, R. Q. Pino, J. Poniachik et al., "Endoscopic band ligation of bleeding dieulafoy lesions: the best therapeutic strategy," Endoscopy, vol. 38, no. 2, pp. 193-194, 2006.

[27] D. Avlan, A. Nayci, E. Altintaş, E. Çingi, O. Sezgin, and S. Aksöyek, "An unusual cause for massive upper gastrointestinal bleeding in children: Dieulafoy's lesion," Pediatric Surgery International, vol. 21, no. 5, pp. 417-418, 2005.

[28] M. J. Morowitz, R. Markowitz, B. M. Kamath, and D. von Allmen, "Dieulafoy's lesion and segmental dilatation of the small bowel: an uncommon cause of gastrointestinal bleeding," Journal of Pediatric Surgery, vol. 39, no. 11, pp. 1726-1728, 2004.

[29] C. Lilje, P. Greiner, U. N. Riede, J. Sontheimer, and M. Brandis, "Dieulafoy lesion in a one-year-old child," Journal of Pediatric Surgery, vol. 39, no. 1, pp. 133-134, 2004.

[30] G. J. Pitcher, D. M. G. Bowley, G. Chasumba, and M. Zuckerman, "Life-threatening haemorrhage from a gastric Dieulafoy lesion in a child with haemophilia," Haemophilia, vol. 8, no. 5, pp. 719-720, 2002.

[31] T. Owaki, C. Kusano, M. Ojiro, and T. Aikou, "Massive bleeding from Dieulafoy's lesion of the small intestine in a child - therapy for the bleeding from gastrointestinal tract out of endoscopic observation," Digestive Surgery, vol. 19, no. 4, pp. 321-324, 2002.

[32] D. Blecker, M. Bansal, R. L. Zimmerman et al., "Dieulafoy's lesion of the small bowel causing massive gastrointestinal bleeding: two case reports and literature review," American Journal of Gastroenterology, vol. 96, no. 3, pp. 902-905, 2001.

[33] R. J. Guy, E. S. W. Ang, K. C. Tan, and C. B. S. Tsang, "Massive bleeding from a Dieulafoy-like lesion of the rectum in a burns patient," Burns, vol. 27, no. 7, pp. 767-769, 2001.

[34] J. A. Stockwell, H. A. Werner, and L. S. Marsano, “Dieulafoy's lesion in an infant: a rare cause of massive gastrointestinal bleeding," Journal of Pediatric Gastroenterology and Nutrition, vol. 31, no. 1, pp. 68-70, 2000.

[35] T. L. Miko and V. A. Thomázy, "The caliber persistent artery of the stomach: a unifying approach to gastric aneurysm, Dieulafoy's lesion, and submucosal arterial malformation," Human Pathology, vol. 19, no. 8, pp. 914-921, 1988.

[36] H. Alis, O. Z. Oner, M. U. Kalayci et al., "Is endoscopic band ligation superior to injection therapy for Dieulafoy lesion?" Surgical Endoscopy and Other Interventional Techniques, vol. 23, no. 7, pp. 1465-1469, 2009.

[37] A. Hoffman, A. Kunert, A. Lahat, A. Volkov, O. Zmora, and D. Rosin, "Laparoscopic resection of gastric dieulafoy lesion 
following preoperative tattooing," Israel Medical Association Journal, vol. 13, no. 3, pp. 187-188, 2011.

[38] K. F. Murray, R. W. Jennings, and V. L. Fox, "Endoscopic band ligation of a Dieulafoy lesion in the small intestine of a child," Gastrointestinal Endoscopy, vol. 44, no. 3, pp. 336-338, 1996.

[39] A. Hokama, R. Ikema, K. Hanashiro, F. Kinjo, and A. Saito, "Endoscopic hemoclipping for duodenal Dieulafoy's lesion," American Journal of Gastroenterology, vol. 91, no. 11, p. 2450, 1996.

[40] J. D. Tooson, L. S. Marsano, and L. K. Gates Jr., "Pediatric rectal Dieulafoy's lesion," American Journal of Gastroenterology, vol. 90, no. 12, pp. 2232-2233, 1995.

[41] H. L. Karamanoukian, D. T. Wilcox, E. I. Hatch, R. Sawin, and P. L. Glick, "Dieulafoy's disease in infants," Pediatric Surgery International, vol. 9, no. 8, pp. 585-586, 1994. 


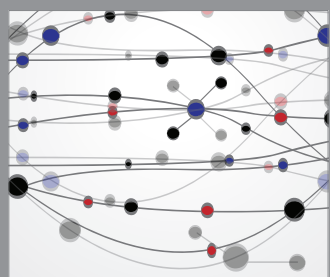

The Scientific World Journal
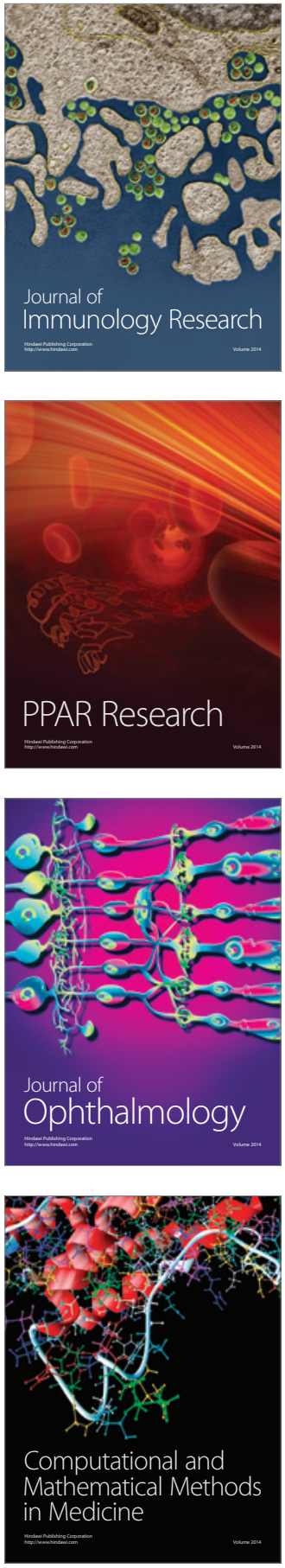

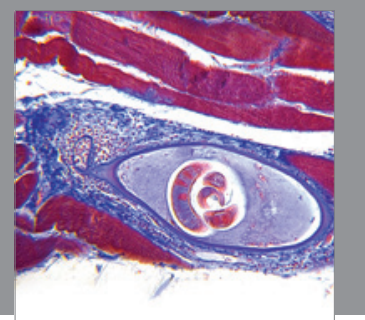

Gastroenterology

Research and Practice
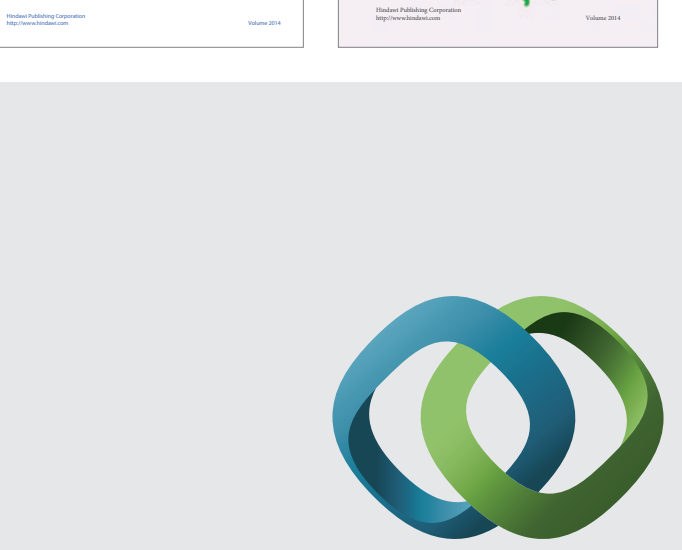

\section{Hindawi}

Submit your manuscripts at

http://www.hindawi.com
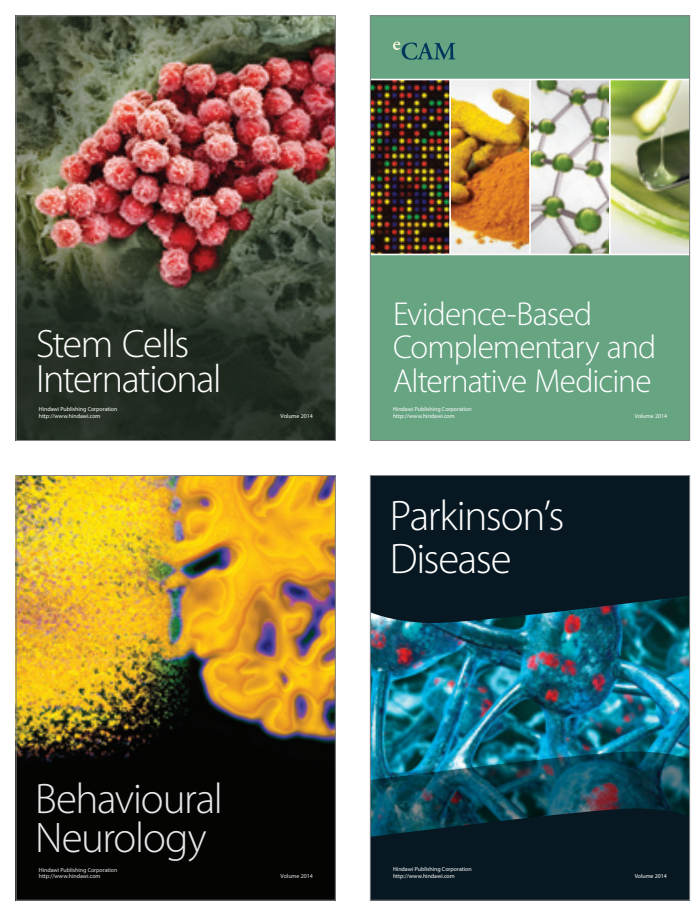

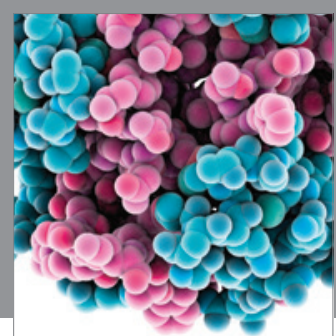

Journal of
Diabetes Research

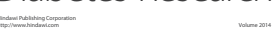

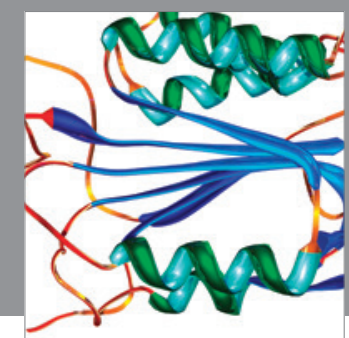

Disease Markers
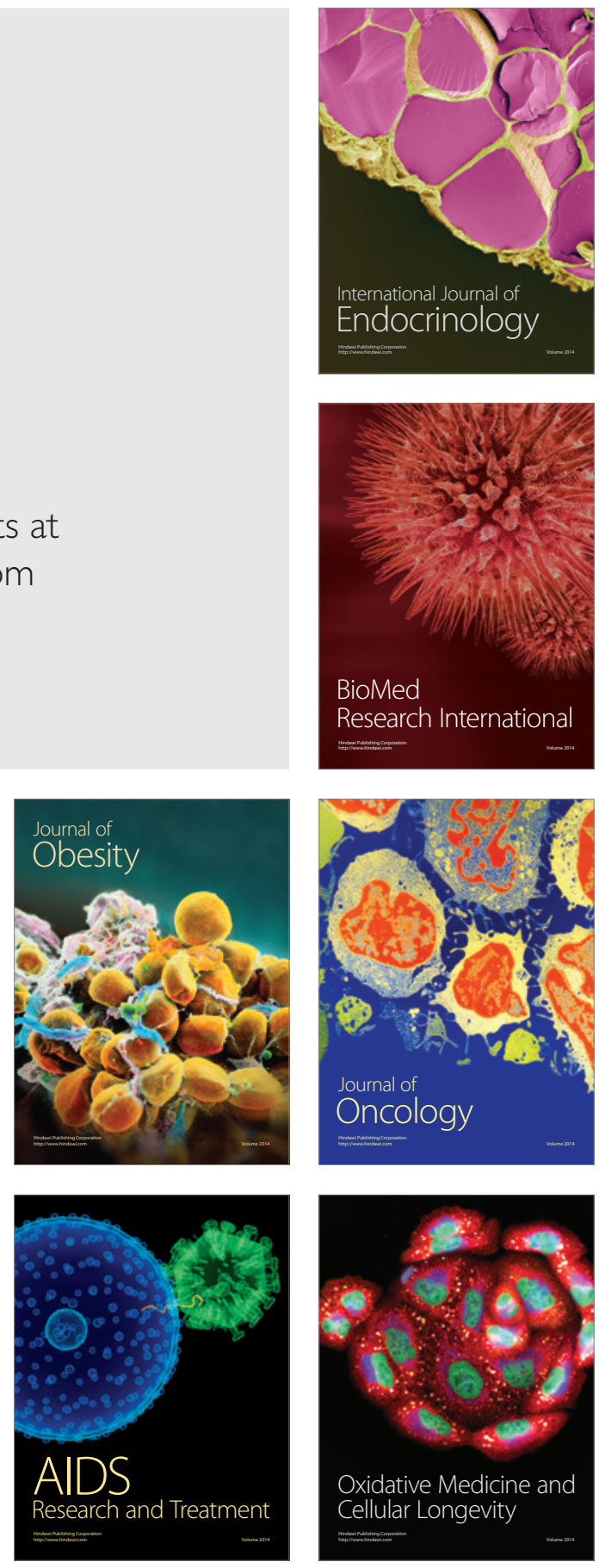